\title{
On the transport of magnetic fields by solar-like stratified convection
}

\author{
S. B. F. Dorch ${ }^{1}$ and $\AA$. Nordlund ${ }^{2}$ \\ 1 The Royal Swedish Academy of Sciences, Stockholm Observatory, 13336 Saltsjöbaden, Sweden \\ e-mail: dorch@astro.su.se \\ 2 The Niels Bohr Institute for Astronomy, Physics and Geophysics, Juliane Maries Vej 30, 2100 Copenhagen $\varnothing$, \\ Denmark \\ e-mail: aake@astro.ku.dk
}

Received 23 June 2000 / Accepted 26 September 2000

\begin{abstract}
The interaction of magnetic fields and stratified convection was studied in the context of the solar and late type stellar dynamos by using numerical 3D MHD simulations. The topology of stratified asymmetric and over-turning convection enables a pumping mechanism that may render the magnetic flux storage problem obsolete. The inclusion of open boundary conditions leads to a considerable flux loss unless the open boundary is placed close to the physical boundary. Simulations including solar-like latitudinal shear indicates that a toroidal field of several tens of kilo-Gauss may be held down by the pumping mechanism.
\end{abstract}

Key words. Sun: MHD - magnetic fields - stratified convection - activity-rotation: stars - magnetic fields

\section{Introduction}

Magnetic fields play an important rôle for the formation of the spectra of active late type G, K and M dwarf stars (e.g. Schrijver \& Rutten 1987; Rutten et al. 1989; Johns-Krull \& Valenti 1996). The most studied star in that respect is the Sun. It is generally assumed that solar active regions are manifestations of a strong toroidal flux system, that is generated and anchored deep below the surface of the Sun, possibly in the undershoot layer below the convection zone proper. Toroidal magnetic strands ascend through the convection zone because of buoyancy and the on the average super-adiabatic stratification. The much weaker poloidal field is assumed to be generated in the convection zone from this toroidal field by a cyclonic effect.

In the mean field dynamo context, the mechanism that takes care of communicating the poloidal field back to the region where the toroidal field is generated, is assumed to be a diffusive coupling of the regions where the $\omega$ effect and the cyclonic $\alpha$-effect operate (e.g. Parker 1993). Choudhuri \& Dikpati (1995) have shown that meridional circulation may also couple the two regions, if the time scale of the circulation is shorter than the diffusive time scale, and if the circulation is such that the flow is equatorward at the bottom of the convection zone. The modeling by diffusive coupling of the regions may be considered to be somewhat unsatisfactory because it relies on a rather

Send offprint requests to: S. B. F. Dorch ad hoc approach and the meridional circulation approach is equally unsafe since it is not well observed - at best the amplitudes and directions of the flow are indicated (van Ballegooijen 1998).

The question of how the poloidal field may return to the region where the generation of the toroidal field supposedly takes place is related to the well-known "buoyancy dilemma" and the generally assumed solution to this problem: any magnetic field in the convection zone will escape because of its buoyancy but a magnetic field may be stored in the stably stratified region below the convection zone; i.e., in the undershoot layer. In that scenario the convection zone is considered to be a passive one-dimensional medium while the magnetic fields are treated more or less as solid objects that move in it. It is conceivable, however, that some kind of balance may occur between the drag of descending plasma and the buoyancy of the magnetic field embedded in the plasma.

Along this line of thinking Drobyshevski \& Yuferev (1974) proposed that a downward "topological pumping" of the magnetic field could be occurring, because of the asymmetric nature of the topology of $3 \mathrm{D}$ convective flows, i.e. that they consist of networks of descending material embedding regions of ascending material. They investigated the kinematic case at low magnetic Reynolds number by assuming an incompressible simple geometrical velocity flow pattern. Criticism by Parker (1975) made Drobyshevski et al. (1980) redo the experiments 
with a more suitable upper boundary condition. Arter et al. (1982), Arter (1983) and Galloway \& Proctor (1983) extended the work to higher magnetic Reynolds numbers and several different compressible flows. They found that the magnetic energy did indeed increase at the bottom of the domain.

During the last decade several groups have performed more detailed magneto-convective numerical simulations (e.g. Hurlburt \& Toomre 1988; Brandenburg et al. 1990; Jennings et al. 1992; Nordlund et al. 1992; Hurlburt et al. 1994; Nordlund et al. 1994; Brandenburg et al. 1996). Moreover, numerical simulations of stratified convection have shown that trace particles initially placed in a horizontal layer of a highly stratified model on the average are transported downwards, as a result of the asymmetric topology of stratified convection (Stein \& Nordlund 1989). In the context of magnetic fields such a tendency for downwards transport of magnetic fields has also been seen in convective dynamo simulations (Nordlund et al. 1992; Brandenburg et al. 1996). Most recently Tobias et al. (1998) have investigated this effect using their standard method for studying stellar convection and Mcleod (1998) presented speculations along the same line of thought that shall be followed here.

\section{Model}

The objective of the numerical experiments presented here is to study the interaction of magnetic fields and solarlike stratified over-turning convection and differential rotation. The model of the Sun is a "local box" model of a convectively unstable layer (henceforth referred to as the "convection zone") sandwiched between two stable layers. In order to circumvent problems associated with the very disparate thermal and dynamical time scales, the model has a much higher luminosity than the Sun, and all variables are scaled accordingly. To compare with solar values, the results must be re-scaled as follows. With a flux scale of $f_{\mathrm{scl}}=310^{5}$ times the solar flux, the velocity scale factor becomes $u_{\mathrm{scl}}=f_{\mathrm{scl}}^{1 / 3} \sim 67$, the temperature fluctuation scale factor $t_{\mathrm{scl}}=u_{\mathrm{scl}}^{2} \sim 4.510^{3}$, and the magnetic field strength scale factor $b_{\text {scl }}=u_{\text {scl }}$. For convenience, the variables in the model are given in units of time $u_{\text {time }}=10^{3} \mathrm{~s}$, length $u_{\text {length }}=1 \mathrm{Mm}$, and density $u_{\rho}=1 \mathrm{~g} \mathrm{~cm}^{-3}$. A Kramers' opacity scaled inversely with the scale factor for the total luminosity is adopted. This ensures that the boundary between the stable layer and the convection zone is at about the same depth in the model as it is in the Sun.

\subsection{Equations}

The full resistive and compressible MHD-equations are solved using the staggered mesh method by Galsgaard and others (e.g. Galsgaard \& Nordlund 1997; Stein \& Nordlund 1989; Nordlund et al. 1992, 1994):

$$
\frac{\partial \rho}{\partial t}=-\nabla \cdot \rho \boldsymbol{u}
$$

$$
\begin{aligned}
\frac{\partial \rho \boldsymbol{u}}{\partial t} & =-\nabla \cdot(\rho \boldsymbol{u} \boldsymbol{u}-\tau)-\nabla P+\boldsymbol{F}_{\mathrm{g}}+\boldsymbol{F}_{\text {Lorentz }}+\boldsymbol{F}_{\mathrm{ext}} \\
\frac{\partial \boldsymbol{B}}{\partial t} & =-\nabla \times \boldsymbol{E} \\
\mu_{0} \boldsymbol{j} & =\nabla \times \boldsymbol{B} \\
\boldsymbol{E} & =\eta \boldsymbol{j}-\boldsymbol{u} \times \boldsymbol{B} \\
\frac{\partial e}{\partial t} & =-\nabla \cdot(e \boldsymbol{u})+P(\nabla \cdot \boldsymbol{u})+Q_{\mathrm{rad}}+Q_{\mathrm{visc}}+Q_{\mathrm{Joule}}
\end{aligned}
$$

where $\rho$ is the mass density, $\boldsymbol{u}$ the velocity, $\tau$ the viscous stress tensor, $P$ the gas pressure, $\boldsymbol{B}$ the magnetic field density, $\boldsymbol{j}$ the current density, $\boldsymbol{E}$ the electric field, $\eta$ the magnetic diffusivity, and $\mu_{0}$ is the magnetic vacuum permeability. In the momentum Eq. (2) $\boldsymbol{F}_{\mathrm{g}}=\rho \boldsymbol{g}$ is the gravitational force, $\boldsymbol{F}_{\text {Lorentz }}=\boldsymbol{j} \times \boldsymbol{B}$ is the Lorentz force and $\boldsymbol{F}_{\text {ext }}$ is the sum of other forces associated with the rotation to be discussed below (Sect. 2.4). The gravitational acceleration $\boldsymbol{g}$ is along the $x$-direction (equivalent to the radial direction). Furthermore $e$ is the internal thermal energy, $Q_{\text {visc }}$ is the viscous heating, $Q_{\text {Joule }}$ is the Joule heating, and $Q_{\mathrm{rad}}=\nabla \cdot(K \nabla T)$ is the radiative heating (cooling) where $K$ is the inverse Kramers' opacity. At the upper boundary an additional expression is used for $Q_{\text {rad }}$ to model surface cooling, see Eq. (7) below.

The code uses a finite difference staggered mesh with 6 th order derivative operators, 5 th order centering operators and a 3rd order time-stepping routine (Hyman 1979).

\subsection{Boundary conditions}

In most stars envelope convection is essentially driven by surface cooling. The entropy contrast at the surface is far larger than that at the bottom of the convection zone, if the convection zone extends over several or many pressure scale heights. To model this situation, without having to actually include all layers up to the solar surface, a simple expression for an isothermal cooling layer at the upper boundary of the model was used:

$Q_{\mathrm{rad}}=-\frac{\left(T-T_{\mathrm{top}}\right)}{\tau_{\mathrm{cool}}} f(x)$,

where $\tau_{\text {cool }}$ is the characteristic cooling time, $T_{\text {top }}$ is the temperature of the cooling layer, and $f(x)$ is a profile function that restricts the effect to a thin surface layer.

Both experiments with closed and open upper boundaries were performed. In order to implement a stable open upper boundary a buffer zone was allocated, where a fiducial electric field is gradually turned on. The sense of the electric field is such that it drags the magnetic field out of the buffer zone, and the magnitude is increased from zero to of the order of $u_{\max } B$, where $u_{\max }$ is the maximum velocity in the buffer zone. A layer that is about 10 grid zones from the numerical upper boundary may thus be considered as the physical open upper boundary. This layer is far below the real boundary of the solar convection zone (even the numerical upper boundary of the model is far below the photosphere). 


\subsection{Initial conditions}

The hydrodynamic part of the initial condition is a snapshot from a well developed stage of a numerical model of the solar convection zone. The initial condition for the magnetic field is given by a unidirectional ("poloidal") sheet that is placed in the middle of the convection zone. The sheet is initially in a state of isentropic pressure equilibrium with the surroundings.

The model has a high degree of stratification with a density contrast of roughly $510^{3}$ in the convection zone alone. The thickness of the undershoot layer in the model is approximately equal to $0.8 \mathrm{H}_{\mathrm{P}}$, where $\mathrm{H}_{\mathrm{P}}$ is the pressure scale height at the bottom of the convection zone. This is much larger than the helioseismological upper limits of $0.1-0.2 \mathrm{H}_{\mathrm{P}}$ (see e.g. Christensen-Dalsgaard 1995). Scaling the extension of the undershoot layer naively in proportion to the velocity (Hooke's law — the retarding force increases approximately linearly with distance), the resulting undershoot thickness, of the order of $0.01 \mathrm{H}_{\mathrm{P}}$, falls well within the upper limits from observations.

\subsection{Latitudinal shear}

Both experiments with and without differential rotation in the convection zone were performed, with the purpose of illustrating the effects of radial and latitudinal shear and of the Coriolis force on the magnetic field. In the rotational cases background differential rotation and the Coriolis force are included by using the force $\boldsymbol{F}_{\text {ext }}$ in the equation of motion Eq. (2)

$\boldsymbol{F}_{\text {ext }}=\boldsymbol{F}_{\text {rot }}+\boldsymbol{F}_{\text {Coriolis }}$

where $\boldsymbol{F}_{\text {Coriolis }}=-2 \rho \boldsymbol{\Omega} \times \boldsymbol{u}$ is the Coriolis force and $\boldsymbol{F}_{\text {rot }}$ is a force designed to induce background rotation (see below).

The differential rotation that is implemented in the model may not be identified as rotation around e.g. one of the horizontal axes. Rather the mapping of the rotation is such that the rotation axis is given by a vector in the "meridional" $(x, y)$ plane pointing towards the north pole, i.e. a rotation vector $\Omega=\left(\Omega_{x}, \Omega_{y}, 0\right)$ given by

$\Omega_{x}=\Omega(x, \theta) \cos \theta$,

$\Omega_{y}=\Omega(x, \theta) \sin \theta$,

where $\Omega=\Omega(x, \theta)$ is a fit to the observed solar angular frequency (from Dziembowski et al. 1989) and $\theta \in[0$ : $2 \pi], \theta(y)=2 \pi y / L_{y}$ where $L_{y}$ is half the size of the box in the latitudinal direction. This choice means that $\Omega$ is parallel to the $x$-axis (the radial direction) at the poles and parallel to the $y$-axis (the latitudinal - or poloidal direction) at the equator.

An azimuthal background velocity corresponding to the observed rotation is implemented by adding a Newtonian term in the equation of motion:

$\boldsymbol{F}_{\text {rot }}=-\frac{\rho}{\tau_{\mathrm{R}}}\left(u_{z}-u_{z}^{0}\right) \hat{\boldsymbol{z}}$, where $\tau_{\mathrm{R}}$ is the time scale of forcing of the background rotation (one turn-over time has proved to be a good choice), $\hat{z}$ is the unit vector in the azimuthal direction, and $u_{z}^{0}=u_{z}^{0}(x, y)$ is the background rotation speed given by

$u_{z}^{0}=-\left(\Omega(x, \theta)-\Omega_{0}\right) R \sin \theta$

where $R$ is a characteristic radius in the convection zone and $\Omega_{0}$ is the angular rotation frequency evaluated at $\theta=\frac{\pi}{4}$.

The differential rotation is then measured relative to the latitude $\pm 45^{\circ}$. In the model that latitude corresponds to the position at fractions of $0.25,0.75,1.25$ and 1.75 of the latitudinal extension of the computational box counting the total length as $2 L_{y}$ (see Fig. 2). At that latitude there is no radial shear since we set the internal solid body rotation equal to the in situ azimuthal speed, so that

$\Omega= \begin{cases}\Omega(x, \theta), & x \geq R \\ \Omega_{0}, & x<R .\end{cases}$

At other latitudes the peak of the radial shear is located in the undershoot layer.

The strength of the background rotation may also be changed by varying the radius $R$ in Eq. (12). Values from 500 to $1400 \mathrm{Mm}$ were used, the higher values in combination with larger values of $\tau_{\mathrm{R}}$, in order to still produce the desired amplitude of the differential rotation.

\section{Results}

Several experiments with varying initial magnetic field strengths, numerical resolutions, and upper boundary conditions were performed. First, results from simulations without rotational effects are discussed (see Table 1), and then results from a simulation including latitudinal shear are reported. Although some of the experiments initially had a maximum field strength of the order of the formal equipartition value, they have been denoted "subequipartition" in Table 1 because the distribution of the kinetic energy density $e_{\mathrm{K}}$ was much broader than the distribution of the magnetic energy density $e_{\mathrm{M}}$, i.e. the peak magnetic energy density was smaller than the peak kinetic energy density even though the most likely value of $e_{\mathrm{M}}$ was similar to that of $e_{\mathrm{K}}$.

A moderate number of grid points were used in these experiments (a few experiments were also ran at higher resolutions, up to $145 \times 128^{2}$, to check for resolution effects). The advantage of performing relatively small numerical experiments, is that it is possible to perform a larger number of experiments, with different setups and with a variety of parameter values. Since it is impossible, with the limits of currently available computer power to accurately reproduce solar conditions, it is necessary to experiment with trade-offs between various constraints.

\subsection{The rotation-less case}

Figure 1 shows two sets of magnetic field isosurfaces at two different instants in time for an experiment with 
Table 1. Summary of five experiments not including rotational effects

\begin{tabular}{ccccc}
\hline Experiment & Initial field & Plasma $\beta$ & Grid points & Top boundary \\
\hline$[\mathrm{i}]$ & Sub-equipartition & $210^{3}$ & $78 \times 64 \times 64$ & Closed \\
[ii] & Kinematic & $210^{5}$ & $78 \times 64 \times 64$ & Closed \\
{$[$ iii] } & Sub-equipartition & $210^{3}$ & $63 \times 64 \times 64$ & Open \\
{$[$ iv] } & Super-equipartition & 200 & $63 \times 64 \times 64$ & Open \\
[v] & Super-equipartition & 20 & $63 \times 64 \times 64$ & Open \\
\hline
\end{tabular}
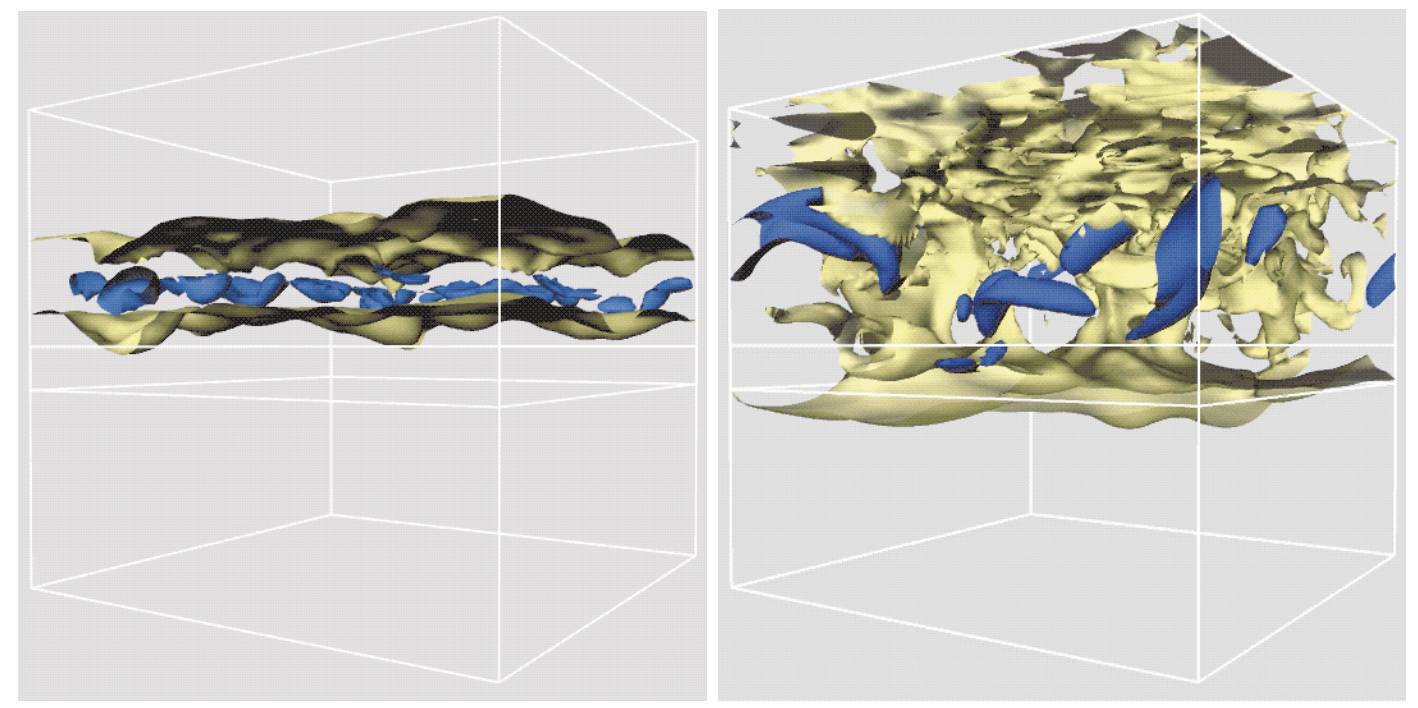

Fig. 1. Snapshot of magnetic field isosurfaces: light gray (yellow), low field strength — dark grey (blue), 10 times higher field strength (colors refer to color versions of the figure, available at http://www.astro.ku.dk/ aake). Left: early in the experiment. Right: at a late time

a sub-equipartition magnetic field (experiment [i], in Table 1): The poloidal sheet of magnetic field initially placed in the middle of the convection zone quickly starts to interact with the convection (Fig. 1, left panel). At a subsequent time (Fig. 1, right panel) the magnetic field more or less fills the whole volume of the convection zone and penetrates into the stable layer below.

Figure 3 shows the horizontally averaged field at 7 equidistant instants of time for experiment [i]: the poloidal sheet is spread out, and the distribution of poloidal magnetic flux settles to a characteristic distribution. The highest (horizontally averaged) poloidal flux density occurs in the overshoot layer, and in these particular experiments a significant fraction of the total flux also resides there. In the real Sun, this fraction may be expected to be much smaller, because the undershoot layer is much thinner there. Given the shape of the distribution, with no particular enhancement in the undershoot layer, it is likely the Sun has a correspondingly smooth distribution, with the majority of the poloidal flux residing inside the convection zone.

The "pumping effect" described in the above takes place because of the topology of the over-turning stratified convection: of all the fluid parcels threaded by magnetic field lines in the initial state about half are initially ascending. However, because of the stratification, most of

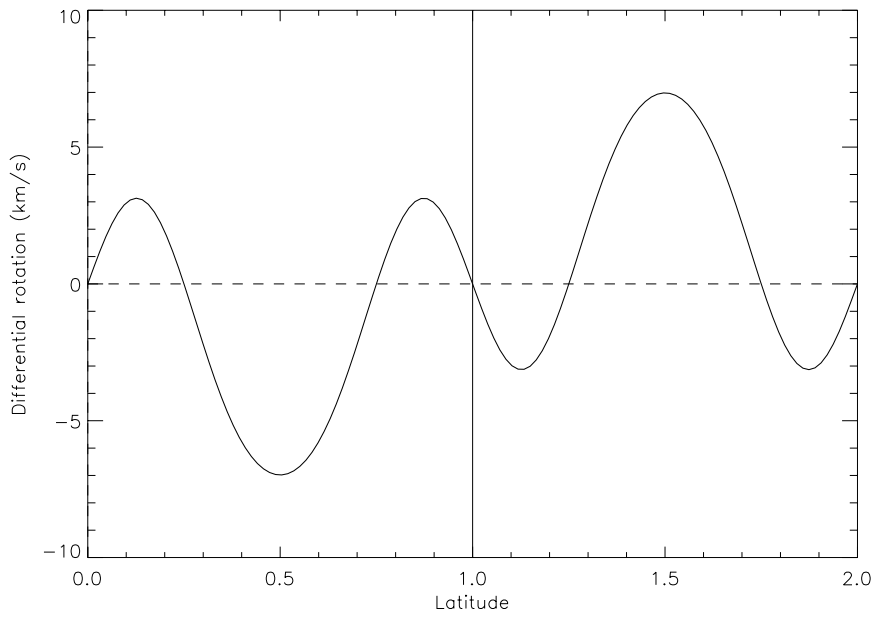

Fig. 2. The background rotational velocity Eq. (12) (in $\mathrm{km} \mathrm{s}^{-1}$ ) as a function of latitude (in units of $\pi$ ); the two equators of the model are at the latitudes 0.5 and 1.5 (corresponding to $\theta=\pi / 2$ and $\theta=3 \pi / 2$, respectively), while the poles are at 0,2 (north pole) and 1.5 (south pole)

the ascending fluid parcels have to over-turn and descend and most of these keep descending down to the bottom of the convection zone (Stein \& Nordlund 1989). The fluid parcels drag the threading field lines along and hence an appreciable fraction of the field is transported downwards. 


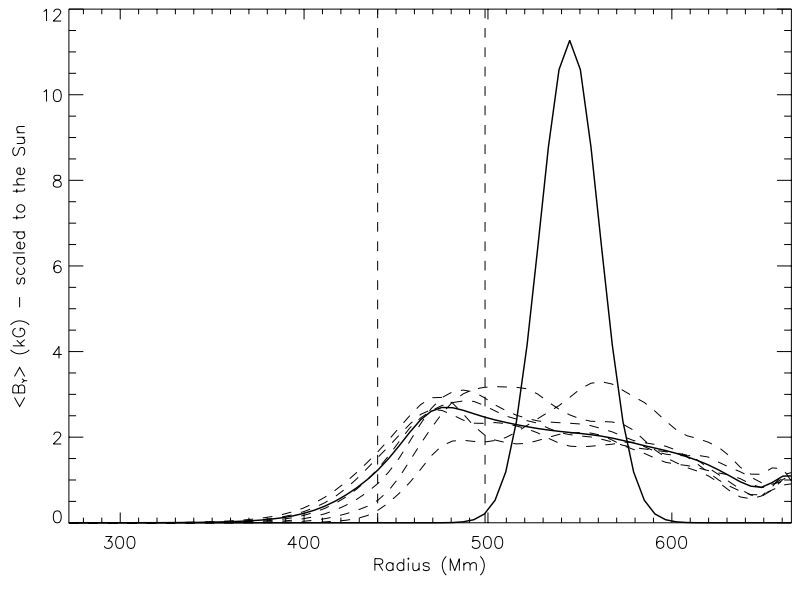

Fig. 3. The horizontally averaged magnetic field $\left\langle B_{y}\right\rangle$ as a function of radius for 7 different times (experiment [i]): $t=$ 0 (initial field - solid curve), 3.3, 6.5, 9.8, 13.0, 16.3 and 19.5 (thin dashed curves), and time-average (solid curve) turn-over times. The approximate bottom and top of the undershoot layer are indicated by two vertical dashed lines

Fragments of the field that are caught in ascending flows are advected upwards. In the experiments with an open upper boundary some of these fragments escape through the top of the computational domain and flux is systematically lost (see the discussion below).

Even in the cases of super-equipartition fields (e.g. experiments [iv] and [v]) the magnetic field is pushed downwards in the initial phases until it reaches the undershoot layer where the kinetic energy flux decreases. Because the overall magnetic flux decreases through flux loss at the surface the magnetic field eventually enters a state in which it is below equipartition.

In the case of an initial field below equipartition the pumping effect is - not surprisingly - even more pronounced than for the super-equipartition fields: Fig. 4 shows the results for three cases where the magnetic field was below equipartition (experiments [i], [ii] and [iii]) and the dynamics thus were dominated by the convective motions. The average magnetic field is distributed over the entire convection zone, with maximum (horizontally averaged) flux density in the undershoot layer.

The radial distribution of the magnetic flux present in the convection zone is not very different for the cases with a closed upper boundary (left panel in Fig. 4) and the cases with an open upper boundary (right panel in Fig. 4), but the total amount of magnetic flux is rapidly reduced during the first few turn-over times, when there is a significant loss of poloidal flux through the upper boundary. This does not influence the distribution of flux over depth much, however, it only influences the amount of flux that is available for distribution.

The flux loss in the models with an open upper boundary is strongly exaggerated in comparison to the Sun. The real flux loss may be expected to be significantly smaller than in the models with open upper boundaries discussed here: Much of the weak ascending flux must over-turn rather than reach the solar surface since it is embedded in a fluid of which only a tiny fraction $(\sim 0.1 \%$ estimated from mass flux amplitudes) reaches the solar surface. Note that the magnetic field considered is weak and incoherent and does not have sufficient buoyancy to overcome the drag of the fluid motions.

A supplementary numerical experiment confirms the exaggeration of the flux loss: moving the open magnetic boundary four mesh point upwards, corresponding to about 0.6 density scale heights, reduces the flux loss by about $20 \%$. The new boundary (at $\rho=2.510^{-2} \mathrm{~g} \mathrm{~cm}^{-3}$ ) is still over ten density scale heights (sic) away from the real solar boundary (at $\rho \sim 310^{-7} \mathrm{~g} \mathrm{~cm}^{-3}$ ). Almost all of the flux that is still lost in the modified experiment may thus in the case of the real Sun be expected to turn over before reaching the solar surface.

Figure 6 shows the average poloidal field strength for the experiments with an open upper boundary (experiments [iii], [iv] and [v]). The relative reduction in the poloidal flux by the escape of magnetic structures through the upper boundary does not depend much on the field strength, for strengths up to several times formal equipartition values.

The rate of flux loss does depend on the field strength but in a rather counterintuitive way: The stronger the initial field the smaller the initial reduction rate. Naively one might expect that if the field strength is large the higher buoyancy would make the field escape faster. The explanation is that the over-turning of the fluid is slowed down in the cases with a stronger initial poloidal flux sheet. The fragmentation of the sheet is also slower in the case with a stronger field.

The magnetic field in the convection zone rapidly becomes very fragmented: while the field in the beginning is uniformly distributed in the sheet, quickly a picture develops where the field in the convection zone becomes very intermittent, while the field that is pumped into the undershoot layer is much more uniform.

The reason for this difference in topology between the magnetic field in the convection zone and in the undershoot layer is that once the magnetic field has been pumped down into the undershoot layer it is less susceptible to fragmentation, since the motions in the stable layer have a much smaller amplitude and are not as systematic as the motions in the convection zone.

The degree of intermittency and fragmentation of the field in the convection zone depends on the strength of the initial poloidal magnetic sheet. The poloidal magnetic sheet is more stable towards the initial fragmentation if its field is stronger since in that case a larger force is needed to over-power the tension in the poloidal field lines.

\subsection{A case with latitudinal shear}

In what follows, results are presented for a particular simulation that includes shear and the Coriolis force (through the force $\boldsymbol{F}_{\text {ext }}$ in the equation of motion Eq. (2)). 

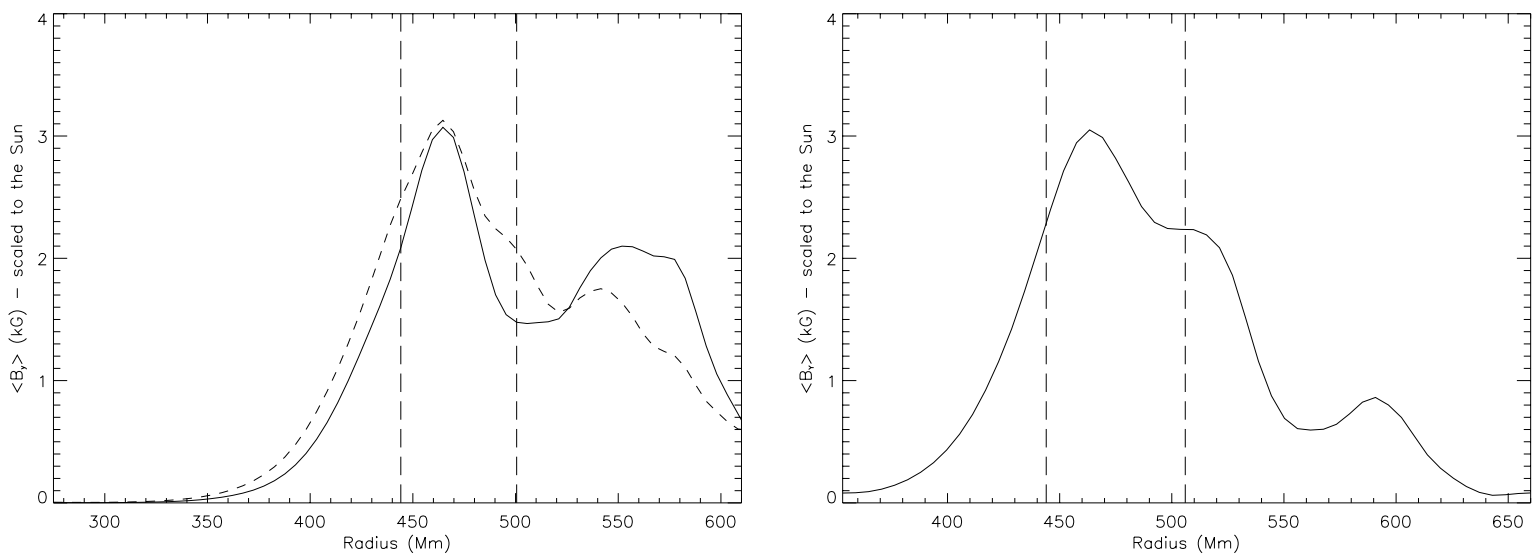

Fig. 4. Horizontal average of the poloidal $B_{y}$ field for two experiments (left panel: solid line, experiment [i] and dashed line, experiment [ii]) that had a closed upper boundary (after 25 and 30 turn-over times respectively), and for experiment [iii] (right panel) that had an open upper boundary (after 18 turn-over times). The amplitudes of the curves have been scaled to have the same maximum as experiment [i] to compare to experiment [i]

The physical size of this experiment was $381 \times 800 \times$ $800 \mathrm{Mm}$ and the numerical resolution $69 \times 135^{2}$ grid points.

The initial condition of a uniform poloidal sheet placed at a certain depth may be said to be rather arbitrary. However, an arbitrary state is both appropriate and useful in this case, since the system rather quickly enters a state that is independent of the initial condition i.e. there is no internal long-time "memory" in the system.

Initially the pumping effect ensures that an on the average poloidal field is injected into the bottom undershoot layer, and after about four turn-over times the poloidal flux density has a maximum below the convection zone of the model.

After an initial transient process where the differential rotation establishes itself and the over-turning convection distorts the poloidal sheet, the system enters a well-developed state, where the magnetic field displays the structure of the differential rotation. At this point, the magnetic energy and the toroidal field strength have increased to a significantly higher level than their initial values (see the two top panels in Fig. 5), and the rms toroidal field is larger than the rms poloidal field. The average poloidal flux has dropped to about half the initial value due to flux loss at the surface (see the bottom two panels in Fig. 5) but this loss of magnetic flux through the upper boundary is somewhat halted after the formation of a large-scale toroidal field.

At first the growth of the toroidal field is fast while the background rotation increases toward the profile determined by Eq. (12) on the time scale $\tau_{\mathrm{R}}$ (cf. Eq. (11)). When the background rotation profile is fully attained by the fluid, the toroidal magnetic field begins to increase linearly, with the rate of increase given by the latitudinal shear and the original poloidal field strength. The structure of the magnetic field directly reflects the latitudinal dependence of the background azimuthal velocity field as a result of the latitudinal shear.
In the well-developed state after the main flux loss has taken place, the dominant magnetic field is a strong toroidal flux system located near the bottom of the convection zone, i.e. it is not pumped into the undershoot layer but it does not escape from the convection zone either: the "center of gravity" of the magnetic field is above the bottom of the convection zone (see Fig. 7).

Figure 8 shows a view of the toroidal flux system: four toroidal flux streets are formed on each side of the equators, in the regions where there is a maximum shear.

The magnetic field just below the convection zone rotates more slowly than the field just above. This is a mechanism that may twist the magnetic field lines that connect across the equator regions.

As the initial poloidal sheet fragments while being wound up, toroidal flux structures leave the convection zone through the open upper boundary. In a rotating frame of reference vertical motions lead to horizontal motions through the action of the Coriolis force. One special case is the rise of flux loops that are rotated so that they emerge tilted with respect to the latitudinal circle, and another is the excitation of meridional circulation, as a result of the transport of angular momentum: in the simulation both of these effects are found.

The surface of the Sun is an extremely important place: not only may flux be lost there, but the surface constitutes a "reconnection region" where vertical field lines are effectively "cut over", and where the remaining "stubs" may be advected passively by the horizontal surface motions. Field lines of course actually continue into the corona and either connect back into the photosphere or connect out into interplanetary space. However, reconnection is observed to proceed so rapidly in the corona and the mass density is so low there, that from the point of view of the sub-surface dynamics the connections above the surface are of little importance.

Figure 9 shows two panels with a small section of the box, with snapshots of the magnetic field, looking down 

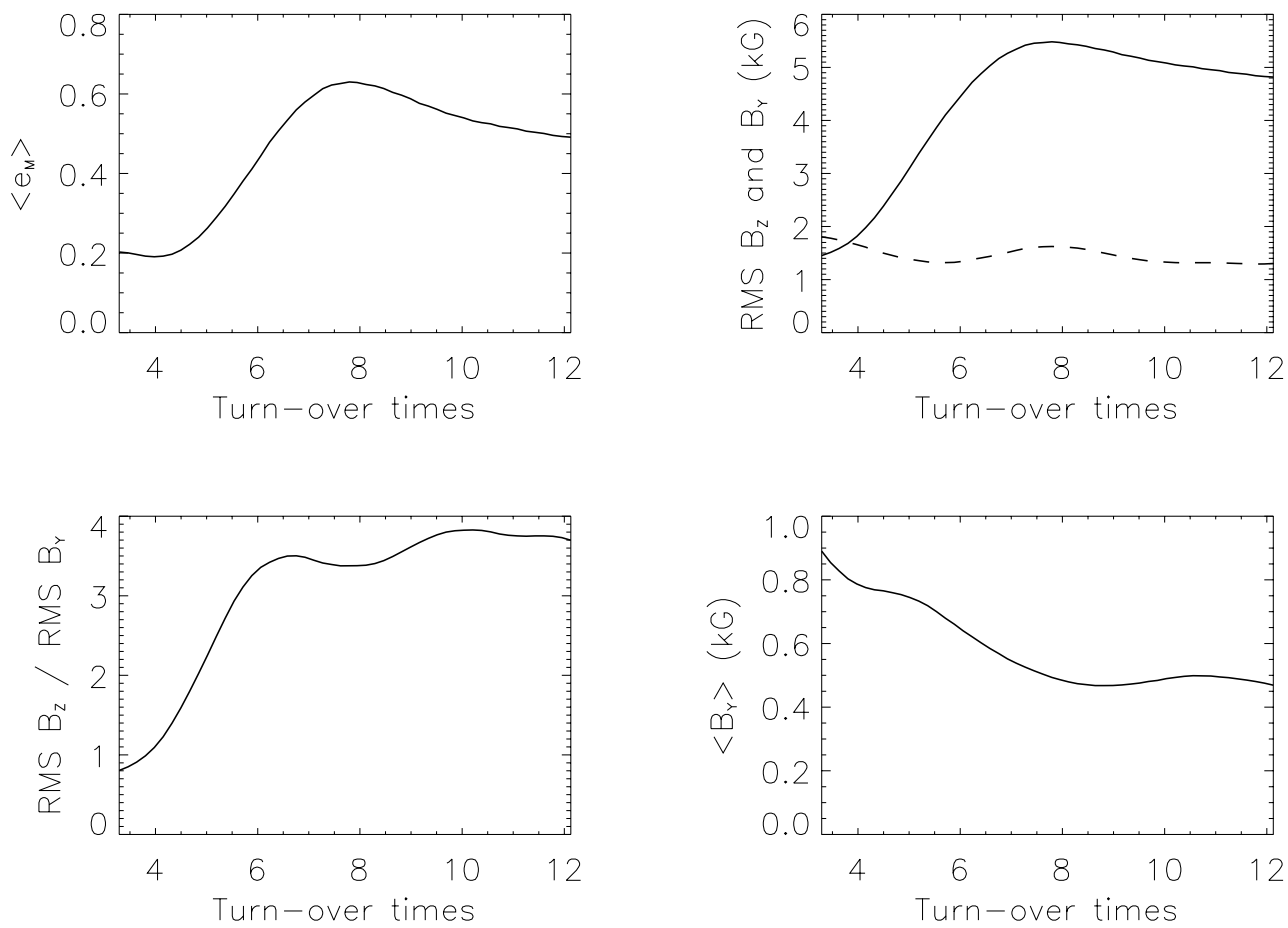

Fig. 5. The case with latitudinal shear: four panels showing the average magnetic energy $\left\langle e_{\mathrm{M}}\right\rangle$, the rms field strengths $B_{z}$ (toroidal field - solid line) and $B_{y}$ (poloidal field - dashed line), the ratio of toroidal to poloidal field, and the average poloidal field $\left\langle B_{y}\right\rangle$ as functions of turn-over times

along the two toroidal flux streets. A magnetic flux structure is sticking out through the surface, and is moving towards the equator between the two toroidal flux streets, but at the same time it is connected through a subsurface structure to one of them. As a result of the drift of the surface-structure, the magnetic field lines that make up the subsurface structure have a tendency to become inclined with respect to the toroidal street to which it connect, and hence a poloidal field component is formed, with opposite polarity relative to the original one.

\section{Discussion and conclusions}

The main conclusions that may be drawn from the study of the interaction of a magnetic sheet with stratified overturning convection in the absence of rotation are the following:

- Stratified convection induces a strong tendency to transport magnetic flux downwards;

- The distribution of horizontally averaged magnetic flux peaks in the undershoot layer, but the bulk of the flux is in the convection zone;

- The magnetic field that resides in the undershoot layer is considerably less fragmented than the magnetic field in the bulk of the convection zone;

- Unless an open upper boundary is placed sufficiently close to the actual solar surface, i.e. at sufficiently low density, there may be a substantial (and exaggerated)

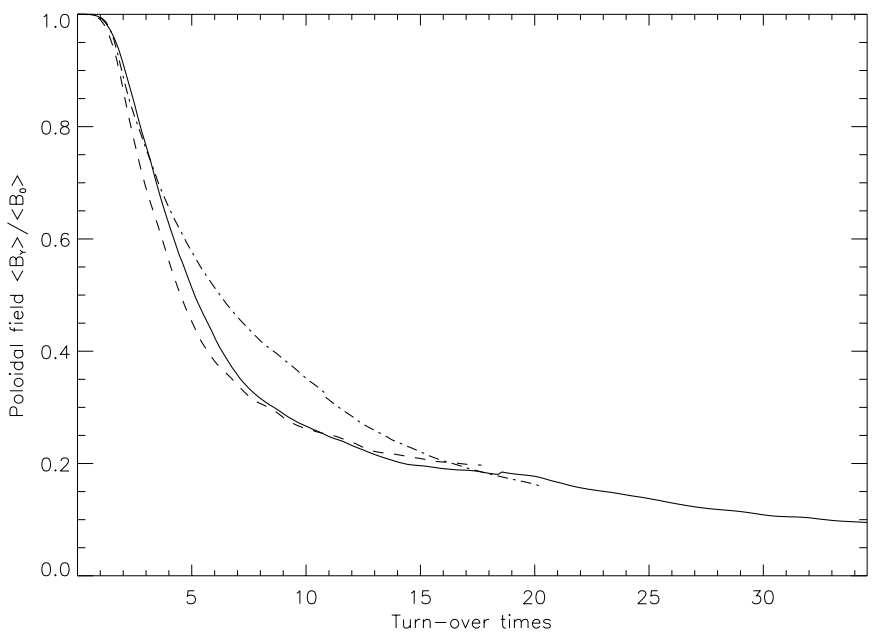

Fig. 6. The normalized average poloidal field strength as function of time in turn-over times for the experiments with an open upper boundary condition: (experiment [iii] dashed curve, experiment [iv] solid curve and experiment [v] dashed-dotted curve)

loss of flux through the open boundary as flux is carried around by the over-turning convection;

- The transport properties (both the downwards transport and the surface flux loss) are quite robust and field strengths well in excess of formal equipartition 


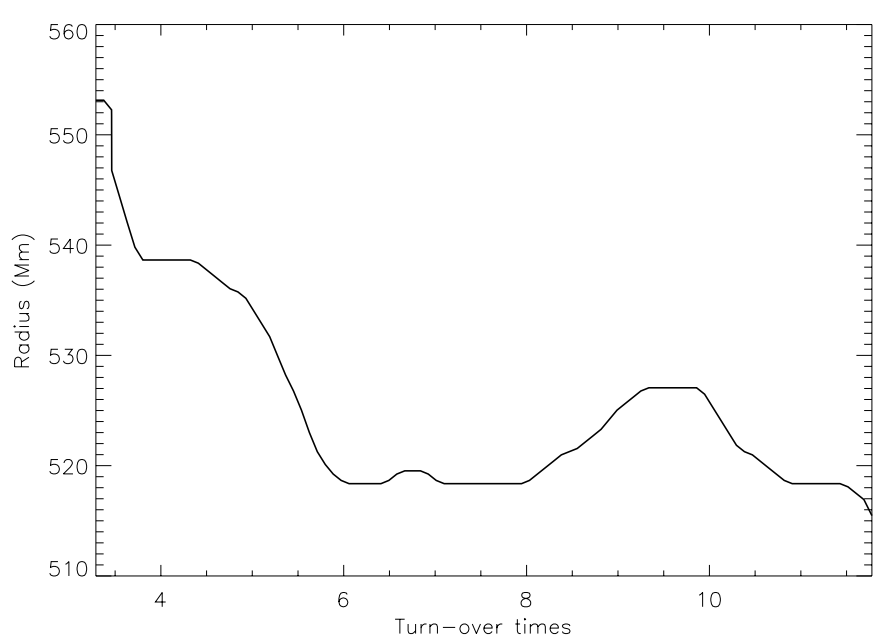

Fig. 7. The depth of the location of the maximum magnetic energy $\left\langle e_{\mathrm{M}}\right\rangle$ as a function of turn-over times

are needed to change the distribution and rates significantly.

Additionally, the results of the simulations including latitudinal shear offer the following conclusions:

- The system rapidly forgets the initial condition and distributes the flux in a generic vertical distribution;

- The latitudinal shear of the differential rotation shapes the magnetic field and creates strong toroidal flux streets located at mid latitudes;

- While magnetic flux indeed is pumped into the undershoot layer, the center of gravity of the magnetic field is above the bottom of the convection zone;

- Magnetic field lines and flux structures penetrating the upper boundary move passively according to the surface motion.

Where do these results leave the "storage problem", i.e. the problem of explaining how the magnetic field can remain stored while being amplified by differential rotation?

The toroidal magnetic field in these simulations reach peak field strengths of a several tens of $\mathrm{kG}$ (scaled to the Sun). The peak field strengths occur near the bottom, but still inside, the convection zone. It is conceivable that emerging flux regions form when buoyancy finally becomes dominant, and that this occurs at field strengths of the order of $100 \mathrm{kG}$, as has been deduced from emergence and tilt patterns by several investigations. Because of numerical limitations we were not able to study that process with the current series of experiments - future experiments with higher resolution and even larger density contrasts are needed here.

Besides offering a clue to the operation of the solar dynamo the results presented here may also contribute to the understanding of magnetic field generation in other late type stars. For example; the fact that no undershoot storage is available in the case of magnetically active fully convective M dwarf stars (e.g. Chabrier \& Baraffe 1997)

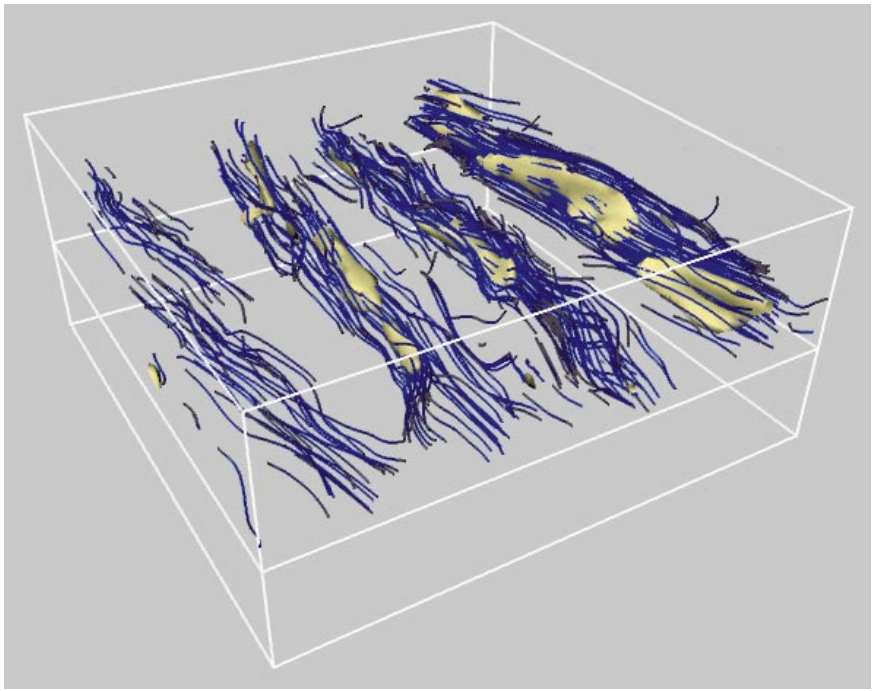

Fig. 8. A snapshot of strong magnetic field lines (blue) and magnetic field isosurfaces (yellow)

is generally considered to be a problem (see e.g. Allard et al. 1997; Küker \& Rüdiger 1999) — this ceases to be a problem in the scenario presented here.

We note that, even though the loss of magnetic flux at the upper boundary is exaggerated in the models presented here, such a loss is certainly a real effect that is important to include, since the Sun is known to loose a considerable amount of toroidal magnetic flux during an activity cycle (e.g. van Ballegooijen 1998).

Lastly we find it important to emphasize that the visualization of "emerging" flux structures and field lines (Fig. 9) illustrates a mechanism first pointed out by van Ballegooijen (1995, private communication) that may be crucial for the reversal of the poloidal field: flux structures that rise and penetrate the surface effectively results in the field lines being "cut" at the surface, with the leading polarity tending to drift towards the equator, and the following polarity tending to drift towards the pole. The result is that subsurface connections between the following polarity of one emerging structure and the leading polarity of another trailing structure may become tilted in the sense opposite to the tilt associated with the normal winding of the field. When such reversed tilts are caught by the differential rotation, they will effectively lead to "unwinding" and reversal of the poloidal field component. This again is an important topic for future studies.

Acknowledgements. This work was supported in part by the Danish Research Foundation, through its establishment of the Theoretical Astrophysics Center. Computing time at the UNI•C computing center was provided by the Danish Natural Science Research Council. SBFD acknowledges support through an EC-TMR grant to the European Solar Magnetometry Network. 

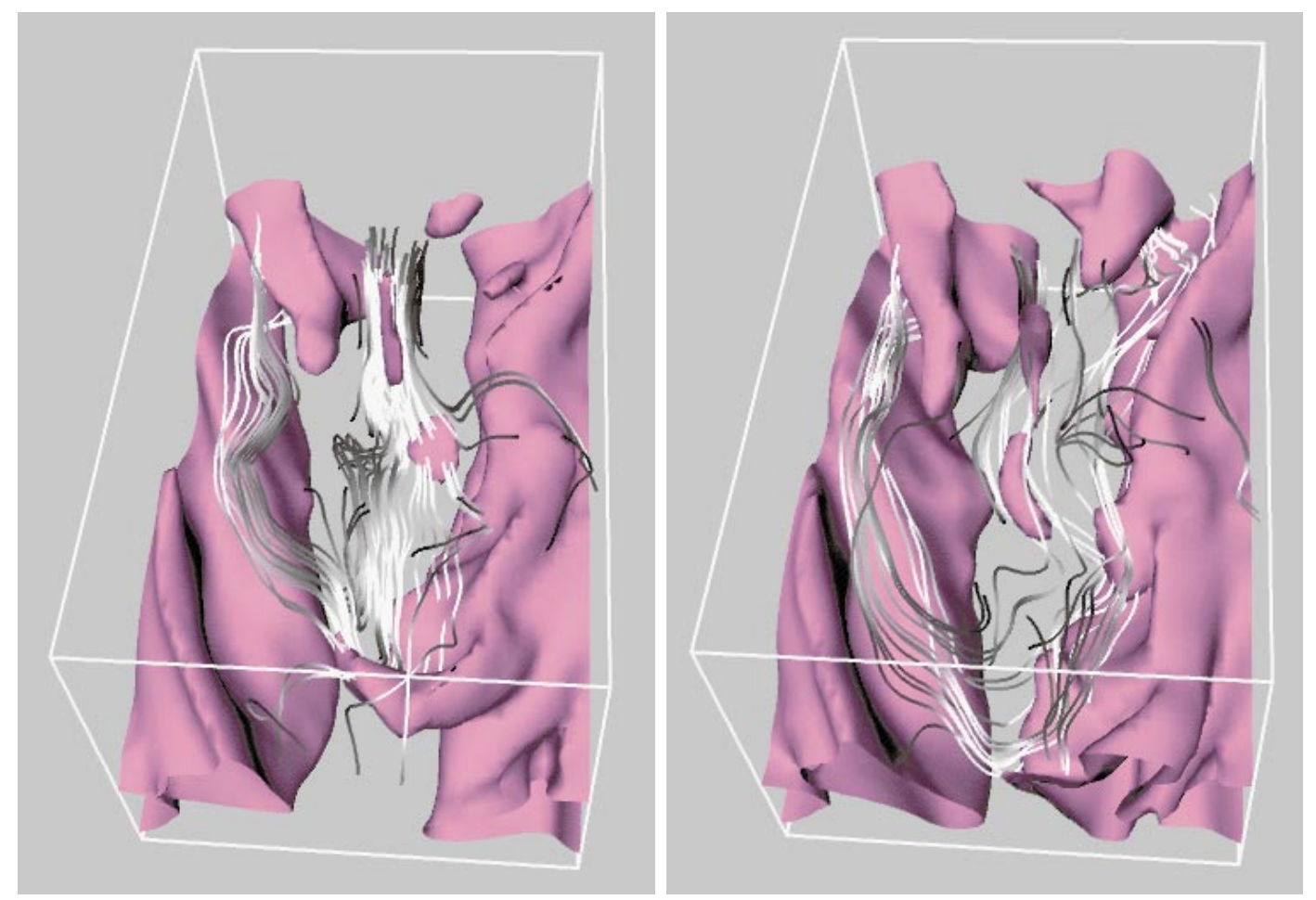

Fig. 9. A view of one quarter of the box, centered at one of the equators, where a magnetic structure is emerging through the surface and is moving towards the equator. Field lines in white and grey (purple) isosurfaces of magnetic field strength. The snapshot to the right is taken $\sim$ half a turnover time later than the one on the left

\section{References}

Allard, F., Hauschildt, P. H., Alexander, D. R., \& Starrfield, S. 1997, ARA\&A, 35, 137

Arter, W. 1983, J. Fluid Mech., 132, 25

Arter, W., Proctor, M. R. E., \& Galloway, D. 1982, MNRAS, 201, 57P

Brandenburg, A., Nordlund, Å., Pulkkinen, P., Stein, R. F., \& Tuominin, I. 1990, A\&A, 232, 277

Brandenburg, A., Jennings, R. L., Nordlund, Å., et al. 1996, JFM, 306, 325

Chabrier, G., \& Baraffe, I. 1997, A\&A, 327, 1039

Choudhuri, A. R., \& Dikpati, M. 1995, A\&A, 303, L29

Christensen-Dalsgaard, J., Monteiro, M. J. P. F. G, \& Thompson, M. J. 1995, MNRAS, 276, 283

Drobyshevski, E., \& Yuferev, V. 1974, J. Fluid Mech., 65, 38

Drobyshevski, E., Lokesnikova, E. N., \& Yuferev, V. 1980, J. Fluid Mech. 101, 65

Dziembowski, W. A., Goode, P. R., \& Libbrecht, K. G. 1989, ApJ, 337, L53

Galloway, D., \& Proctor, M. R. E. 1983, Geophys. \& Astrophys. Fluid Dyn., 24, 109

Galsgaard, K., \& Nordlund, Å 1997, J. Geophys. Res., 102, 219

Hurlburt, N. E., \& Toomre, J. 1988, ApJ, 327, 920

Hurlburt, N. E., Toomre, J., Massaguer, J., \& Zahn, J.-P. 1994, ApJ, 421, 245
Hyman, J. M. 1979, Adv. Comp. Meth. for PDE's—III, ed. R. Vichnevetsky, R. S. Stepleman, Publ. IMACS, 313

Jennings, R., Brandenburg, A., Nordlund, A., \& Stein, R. F. 1992, MNRAS, 259, 465

Johns-Krull, C. M., \& Valenti, J. A. 1996, ApJL, 459, L95

Küker, M., \& Rüdiger, G. 1999, in Stellar dynamos: Nonlinearity and chaotic flows, ed. A. Ferriz-Mas, \& M. Nunez, ASP, 178,87

Mcleod, A. D. 1998, in Numerical Astrophys., ed. S. M. Miyama, et al. 331

Nordlund, Å., Brandenburg, A., Jennings, R. L., et al. 1992, ApJ, 392, 647

Nordlund, A., Galsgaard, K., \&. Stein, R. F. 1994, in Solar Surface Magnetic Fields NATO ASI Ser., ed. R. J. Rutten, \& C. J. Schrijver, 433

Parker, E. N. 1975, ApJ, 202, 523

Parker, E. N. 1993, ApJ, 408, 707

Rutten, R. G. M., Schrijver, C. J., Zwaan, C., Duncan, D. K., \& Mewe, R. 1989, A\&A, 219, 239

Schrijver, C. J., \& Rutten, R. G. M., 1987, A\&A, 117, 143

Stein, R. F., \& Nordlund, Å. 1989, ApJ, 342, L95

Tobias, S., Brummel, N., Clune, T., \& Toomre, J. 1998, ApJ, 502, L177

van Ballegooijen, A. A. 1998, in Synoptic Solar Physics 140 (Kluwer Academic Publishers), 17 